\title{
Experimental Demonstration of Dielectric Structure Based Two Beam Acceleration
}

\author{
Wei Gai, M. E. Conde, R. Konecny, J. G. Power, P. Schoessow, X. Sun \\ and P. Zou
}

High Energy Physics Division, Argonne National Laboratory

Argonne, IL 60439

\begin{abstract}
We report on the experimental results of the dielectric based two beam accelerator (step-up transformer). By using a single high charge beam, we have generated and extracted a high power RF pulse from a $7.8 \mathrm{GHz}$ primary dielectric structure and then subsequently transferred to a second accelerating structure with higher dielectric constant and smaller transverse dimensions. We have measured the energy change of a second (witness) beam passing through the acceleration stage. The measured gradient is $>4$ times the deceleration gradient. The detailed experiment of set-up and results of the measurements are discussed. Future plans for the development of a $100 \mathrm{MeV}$ demonstration accelerator based on this technique is presented.
\end{abstract}

\section{INTRODUCTION}

The development of robust accelerating structures is critical for future high energy accelerators. The concept of using dielectric loaded structure as acceleration structure has been around for many years [1], and in particular there have been a number of proposals to use dielectric loaded structures as collinear wakefield accelerators $[2,3]$. Recently, there have also been proposals to also use dielectric structures as power extraction devices for high frequency rf generation [4]. The simplicity of this method, as well as the relative ease with which parasitic higher order modes can be damped compared to conventional structures[5] operating at comparable frequencies makes this technology an attractive option for future high energy linear colliders.

Another important issue for linear collider development is RF sources. Using bunched train driven dielectric based technology for RF extraction directly from an intense relativistic bunched electron beam has significant advantages since the radiation frequency only depends on the dielectric device geometry. The RF source frequency can be easily tuned to a harmonic of the linac RF frequency with some tuning range (by adjusting the laser spacing for the RF photocathode gun). In fact, at very high frequencies, it can be viewed as a continuous RF source. This RF power is then directly transferred to a second dielectric tube with much higher dielectric constant, compressing the pulse and hence enhancing the acceleration field. This scheme is also called a step-up transformer; the use of separate beam paths allow transformer ratios $>2$ to achieved [4]. By using a multiple drive beam, longer

Submitted to the proceedings of the 9th Advanced Accelerator Concepts Workshop (AAC), Santa Fe, NM, June 10-16, 2000. 


\section{DISCLAIMER}

This report was prepared as an account of work sponsored by an agency of the United States Government. Neither the United States Government nor any agency thereof, nor any of their employees, make any warranty, express or implied, or assumes any legal liability or responsibility for the accuracy, completeness, or usefulness of any information, apparatus, product, or process disclosed, or represents that its use would not infringe privately owned rights. Reference herein to any specific commercial product, process, or service by trade name, trademark, manufacturer, or otherwise does not necessarily constitute or imply its endorsement, recommendation, or favoring by the United States Government or any agency thereof. The views and opinions of authors expressed herein do not necessarily state or reflect those of the United States Government or any agency thereof. 


\section{DISCLAIMER}

Portions of this document may be illegible in electronic image products. Images are produced from the best available original document. 
acceleration distance can also be achieved, thus obtain higher gradient and sustained $S$ T I accelerations than the collinear schemes.

In this paper, we report on the proof of principle experimental results on dielectric loaded two beam acceleration experiment. In the past year, we have completed an upgrade of the Argonne Wakefield Accelerator [6,7] with a parallel witness beam line with a magnetic spectrometer for energy measurement of the witness beam. The typical witness beam energy is about $3.5 \mathrm{MeV}$ with charge of $0.5 \mathrm{nC}$. The step-up transformer experiment is shown in Figure 1 schematically.

\section{Schematic Diagram of the Step-up Transformer experiment}

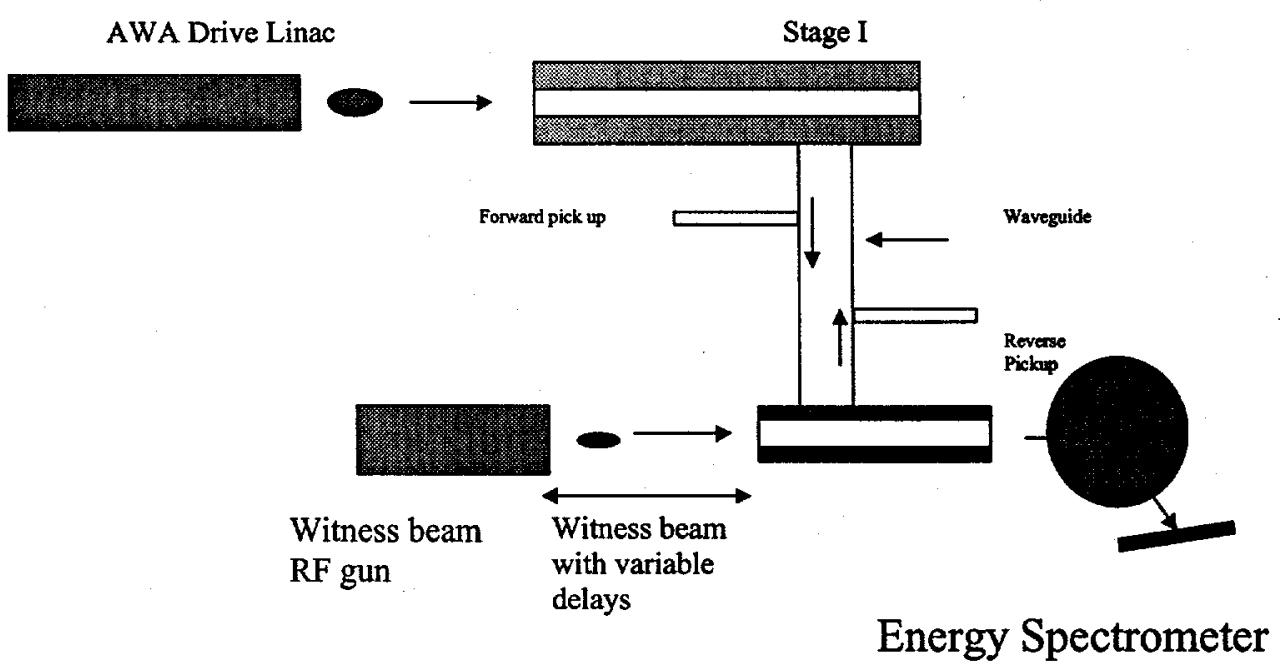

FIGURE 1. Schematic diagram of the two beam acceleration (step-up transformer) experiment. The wakefield generated in Stage I structure is taken out and transferred to a second structure to establish higher acceleration gradient. A less intense witness is injected in the second structure for acceleration experiment. Its energy change depends on the relative delay between the drive and witness beam.

\section{Dielectric Structure Design and Construction}

The structures used for these experiments were designed to demonstrate the physics of dielectric based two beam acceleration. The choice of operating frequency is 7.8 $\mathrm{GHz}$ which is compatible with the drive bunch length available at AWA while also a harmonic of the $1.3 \mathrm{GHz}$ frequency. The device parameters are summarized in Table 1 .

TABLE 1. Parameters for staged dielectric step-up transformer

\begin{tabular}{lcc} 
& Stage I & Stage II \\
\hline Inner Radius a & $6 \mathrm{~mm}$ & $3 \mathrm{~mm}$ \\
Outer radius b & $11.15 \mathrm{~mm}$ & $5.41 \mathrm{~mm}$ \\
Dielectric constant $\varepsilon$ & 4.6 & 20 \\
Group velocity $\beta_{\mathrm{g}}$ & 0.25 & 0.05 \\
$\mathrm{E}_{\mathrm{z}}$ (Max) & $3 \mathrm{MV} / \mathrm{m}$ & $8 \mathrm{MV} / \mathrm{m}$ \\
Designed field step up & & 2.5 \\
Interaction length for a single & $30 \mathrm{~cm}$ & $3 \mathrm{~cm}$ \\
beam & & \\
\hline
\end{tabular}


The structures were constructed in house and cold tested using a HP8510C network analyzer. It was very challenging to achieve good coupling between the stages because of the wave impedance mismatch between the dielectric loaded waveguide and standard rectangular waveguide. This problem was solved by tapering the dielectric inner radius near the coupling slot so it can act as an impedance transformer. The measured transmission from stage I to stage II was $>96 \%$, sufficient for the proof of principle experiment.

\section{Beam Measurement Results}

After the structures were constructed, they were installed in the beamline for experiment using electron beam from the drive gun. In this section we discuss the experimental results.

\section{$R F$ Power Spectrum and Transfer Measurements}

The first experiment conducted was to measure the RF power generated from the stage I dielectric tube. We used a HP spectrum analyzer capable of measurements up to $50 \mathrm{GHz}$. Figure 2 shows the measured spectrum from the forward power flow. It shows that majority of the energy is concentrated near $7.8 \mathrm{GHz}$ as expected. However, a small peak around $8.3 \mathrm{GHz}$ is not expected and further investigation is needed. Expanding the frequency range showed the second deflection mode at 11 $\mathrm{GHz}$ as expected. Due to the finite coupling iris band width, we could not observe the first deflection mode.

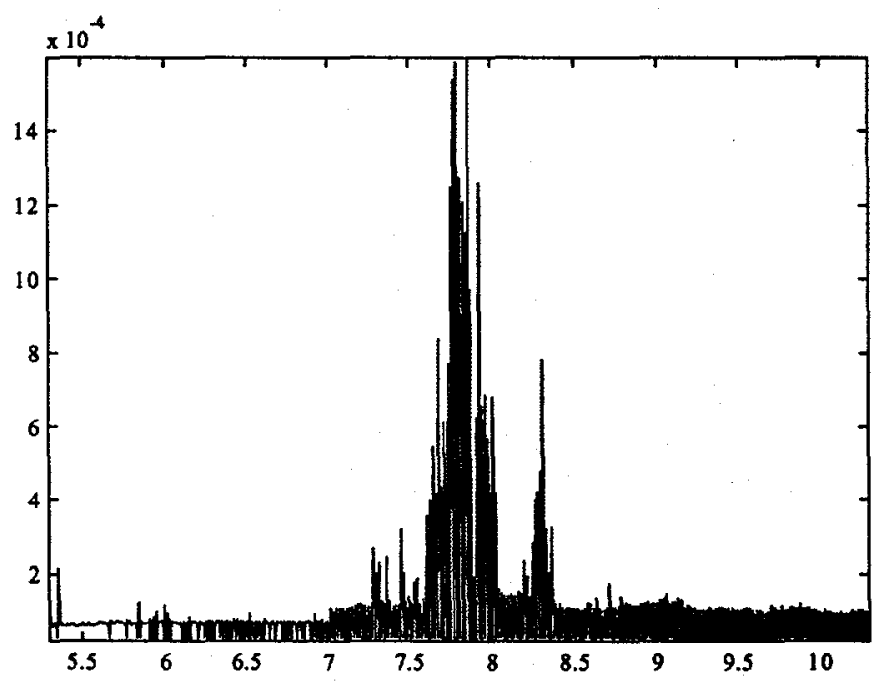

FIGURE 2. Measured spectrum of the beam induced RF signal from the stage I tube. The energy is concentrated around the $\mathrm{TM}_{01}$ fundamental frequency $7.8 \mathrm{GHz}$ as expected.

The RF power flow was also measured between the two stages. In order to do this, a directional coupler was installed in the transfer waveguide. The forward and - reflected RF power signal envelope is detected using a high frequency diode as shown 
in FIG 3. The measured RF pulse width is $2.5 \mathrm{~ns}$, which agrees very well with the prediction based on the dielectric property and structure length. The timing between the forward and reflected power indicates that the small reflection is from the coupling iris of the stage II tube. The estimated reflection coefficient is $<5 \%$, as expected from the bench coupling measurements.
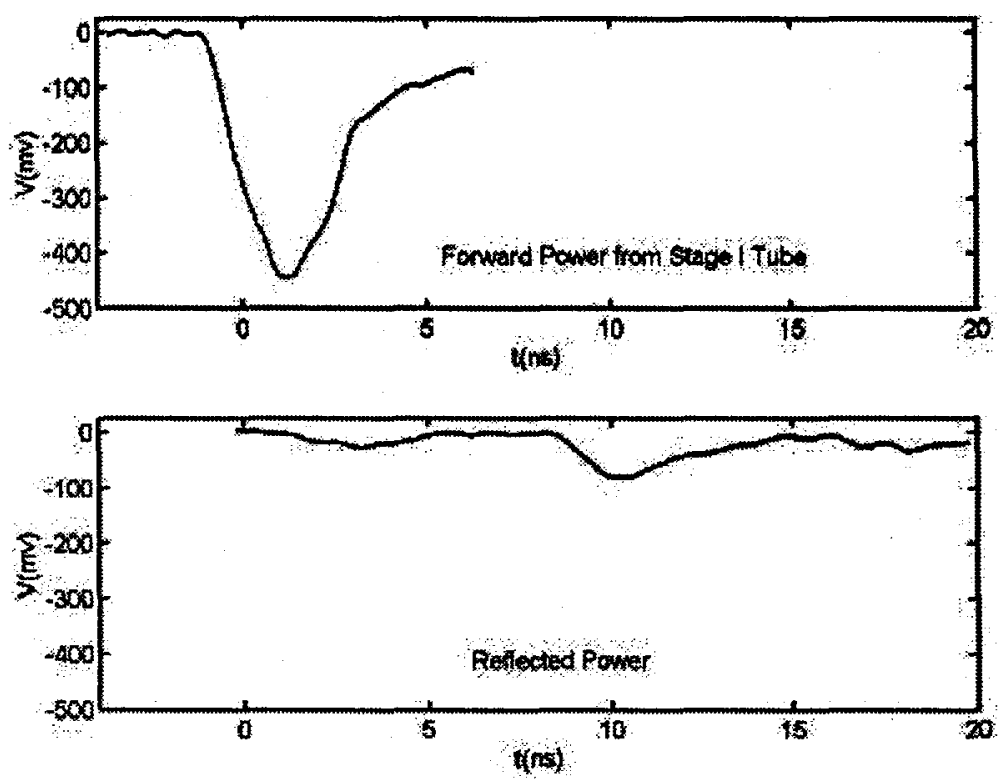

FIGURE 3. Measured forward and reflected RF power envelope from the stage I to stage II tube. The peak power is $4 \mathrm{MW}$. The estimated reflected $\mathrm{RF}$ power is $<5 \%$, corresponding to the bench measurement of the stage I-II coupling.

\section{Acceleration of the Witness Beam in Stage II}

When the witness beam injected into the stage II structure, its energy will change depending on its phase relative to the drive beam. At the AWA, this phase is adjusted by varying simultaneously the laser delay and RF phase to the witness gun [7]. Figure 4 shows the results of a typical measurement. The horizontal axis is the energy (bend) plane of the spectrometer. The top picture is the witness beam imaged on a phosphor screen in the spectrometer focal plane with no drive beam passing through the stage 1 tube. In this experiment, the drive beam charge is $\sim 20 \mathrm{nC}$, which would be expected to generate 1.6 MV/m decelerating gradient in the stage 1 tube (and $\sim 3 \mathrm{MV} / \mathrm{m}$ peak field) as measured indirectly from the forward RF power (4 MW, in Fig 3). The middle picture shows the witness energy at 56 ps nominal delay. (Note the nominal drive-witness delays given below are measured with repect to an arbitrary zero-delay point). The maximum energy gain is about $250 \mathrm{KeV}$ which is corresponding to about $7.5 \mathrm{MeV} / \mathrm{m}$, agreeing well with the prediction as shown in table 1 . Therefore, the field step-up ratio is $>2$ as predicted. Moreover, This also mean the transformer ratio (Maximum accelerating field/maximum decelerating field in driving tube) exceeds 4. This demonstrates experimentally that the wakefield theorem can be violated in a noncollinear geometry devices. When the delay is changed to $-8 \mathrm{ps}$, the witness 
beam experienced maximum decelerating phase of the wakefields. Thus the wavelength in the second stage is $\sim 132 \mathrm{ps}$, which corresponds to $7.8 \mathrm{GHz}$ excitation in stage II with phase velocity c.
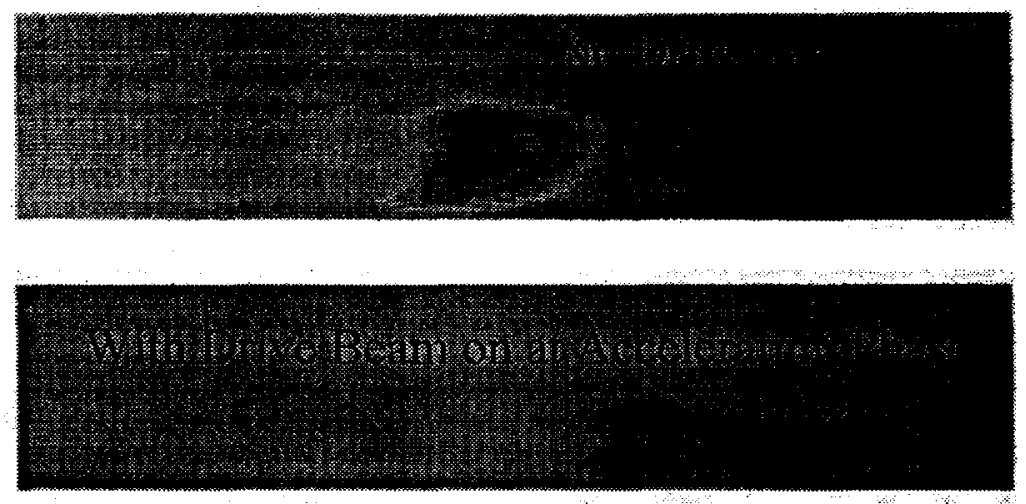

$\mathrm{T}=56 \mathrm{ps}$

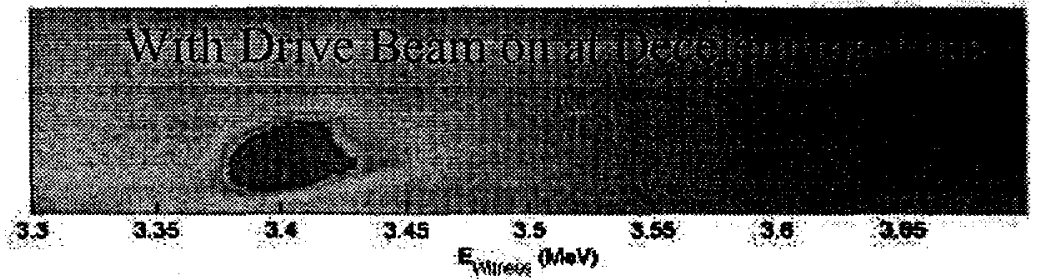

$\mathrm{T}=-8 \mathrm{ps}$

Figure 4. Measured energy change of the witness beam. The top is with drive beam off and middle is with drive beam on and with acceleration phase of beam delay at $56 \mathrm{ps}$. The bottom shows the deceleration.

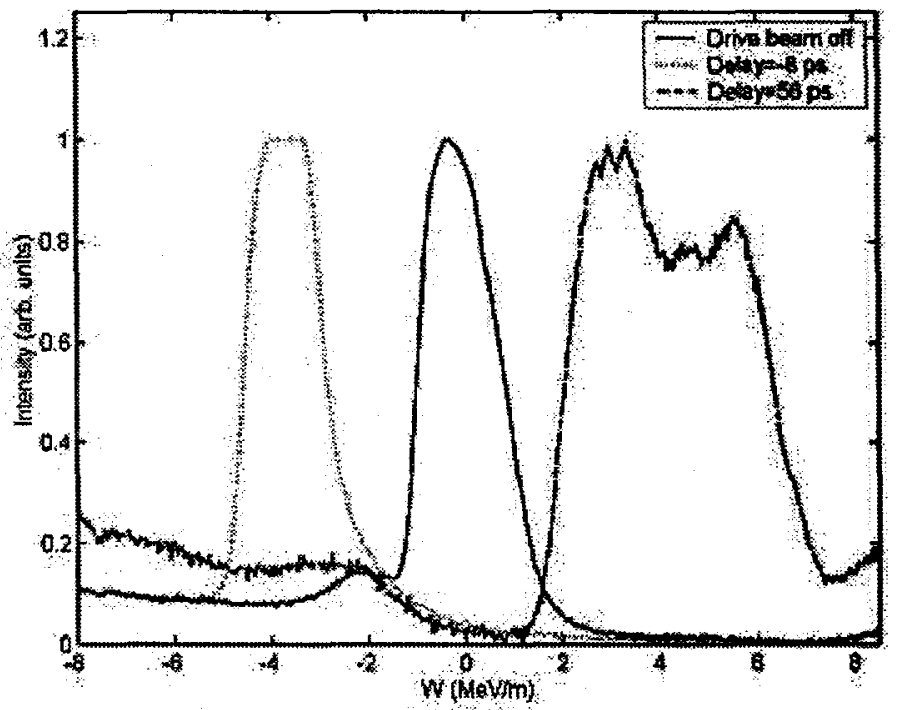

Figure 5. Bend view projections of the data in Figure 4. 
Projections of the beam profile in the energy plane are shown in Figure 5. The measured energy change of the witness beam in the stage II dielectric tube demonstrated the following results: the RF generated is efficiently coupled to the $\mathrm{TM}_{01}$ accelerating mode between the stages. The field gradient in stage II is steppedup by a factor $>2$ with transformer ratio of $>4$. Finally, the efficient coupling technique developed using bench measurements is found to couple to the desired phase velocity for $\mathrm{TM}_{01}$ acceleration mode.

\section{Future Plans}

Although we have successfully tested the step-up transformer concept using the two beam acceleration method, there are still some detailed experiments to be done. In the short term, we will map in detail the accelerating field by adjusting the pulse delay between the drive and witness beams in small increments and observing the change in witness beam energy. Then we will use the multiple drive beam to increase the acceleration distance from $3 \mathrm{~cm}$ to $12 \mathrm{~cm}$ using 4 drive pulses.

The limitating factor in accessing higher acceleration gradients is the drive beam properties, in particular that the current drive beam has relative high emittance and longer bunch length. A further limiting factor is the number of drive bunches produced due to the quantum efficiency of the photocathode. There is major ongoing effort to improve the drive beam properties by constructing a third generation drive gun[8]. The new gun is a $1 \frac{1 / 2}{2}$ cell RF photocathode gun with axial electric field of 80 $-100 \mathrm{MV} / \mathrm{m}$. Based on PARMELA[9] simulations, it will produce much lower emittance (by a factor of 20) and shorter beams $(3-4 \mathrm{ps})$ for intensities of $40-100 \mathrm{nC}$. Another improvement will come from the operating vacuum and cathode upgrades. We intend to replace the current $\mathrm{Mg}$ cathode with a CsTe type high QE cathode. Based on the current available AWA laser system (5 mJ, 4ps @ $248 \mathrm{~nm}$ ), we could produce a pulse train up to 4 pulses with $100 \mathrm{nC}$ each. By using this pulse train we could easily achieve $100 \mathrm{MV} / \mathrm{m}$ acceleration gradient using the same two beam acceleration (step-up transformer) structures used in this experiment. This gun would also produce up to 64 pulses with $40 \mathrm{nC}$ each. By using the same step up transformer, we would not only achieve $100 \mathrm{MV} / \mathrm{m}$, but also could accelerate the beam to $100 \mathrm{MeV}$ in less than a meter. This is the equivalent of powering the second stage tube with external $500 \mathrm{MW}$ RF power source with a $50 \mathrm{~ns}$ pulse length. The new electron gun is expected to be in operation in the fall of year 2000 .

\section{SUMMARY}

Considerable progress has been made towards a demonstration of the dielectric loaded two beam accelerator concept. A proof of principle experiment clarified the associated physics and engineering issues such as RF coupling and acceleration in the correct acceleration mode. With the new AWA electron gun, we will further extend this experiment by using the high current pulse train to provide $>100 \mathrm{MV} / \mathrm{m}$ gradient over an acceleration distance of 1 meter. 


\section{ACKNOWLEDGMENTS}

This work is supported by the US Department of Energy, Division of High Energy Physics, under contract W-31-109-ENG-38.

\section{REFERENCES}

1. G. Flesher and G. Cohn, AIEE Trans. 70, 887 (1951)

2. R. Keinigs, M. Jones, and W. Gai, Part. Accel. 24, 223 (1989)

3. T. B. Zhang, J. Hirshfield, T. Marshall, and N. Hafizi, Phys. Rev. E 56, 4647 (1997)

4. E. Chjonacki, W. Gai, P. Schoessow, and J. Simpson, in Proceedings of Particle Accelerator Conference, edited by L. Lizama and J. Chew (IEEE, San Francisco, 1991), pp. 2557-2559.

5. E. Chjonacki et al, J. Appl. Phys. 69

6. M. E. Conde et al, Phys. Rev. ST Accel. Beams 1, 041302, 1998.

7. M. E. Conde et al, "A High-Charge High-Brightness L-Band Photocathode RF Gun", Submitted to the Proceedings of the ICFA Advanced Accelerator Workshop (Los Angeles, Nov. 1999) edited by J. Rosenzweig, World Scientific Publisher, 2000

8. J. G. Power and M. E. Conde, Rev. Sci. Instrum. 69, 1295 (1998).

9. SUPERFISH and PARMELA, Los Alamos National Laboratory Report No. LA-UR-96-1834, 1997; Report No. LA-UR-96-1835, 1996. 